NASA Technical Memorandum 86188

\title{
Spectral Constraints on Models of Gas in Clusters of Galaxies
}

Mark J. Henriksen and Richard F. Mushotzky

MAY 1985

\section{LIBRARY COPY

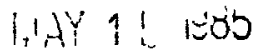 \\ LANGLEY RESEARCH CENTER LIBRARY, NASA HAMPTON, VIRGINIA}


NASA Technical Memorandum 86188

\title{
Spectral Constraints on Models of Gas in Clusters of Galaxies
}

\author{
Mark J. Henriksen \\ University of Maryland \\ College Park, Maryland \\ Richard F. Mushotzky \\ Goddard Space Flight Center \\ Greenbelt, Maryland
}

\section{N/SA \\ National Aeronautics \\ and Space Administration}

Scientific and Technical

Information Branch 
SPECTRAL CONSTRAINTS ON MODELS OF GAS IN CLUSTERS OF GALAXIES

Mark J. Henriksen ${ }^{1}$ and Richard F. Mushotzky

Laboratory for High Energy Astrophysics

NASA/Goddard Space Flight Center

Greenbelt, Maryland 20771 IISA

ABSTRACT. HEAO 1-A2 spectra of clusters of galaxies are used to determine the temperature profile which characterizes the $X$-ray emitting gas. Strong evidence of non-isothermality is found for the Coma, A85, and Al795 clusters. Properties of the cluster potential which binds the gas are calculated for a range of model parameters. The typical binding mass, if the gas is adiabatic, is $2-4 E 14$ solar masses and is quite centrally concentrated. In addition, the Fe abundance in Coma is .26 \pm .06 solar, less than the typical value (.5) found for rich clusters. Dur results for the gas in Coma may imply a physical description of the cluster which is quite different from what was previously believed.

\section{INTRODUCTION}

We modeled the $X$-ray spectra from 10 clusters of galaxies observed with the HEAO 1-A2 medium and high energy detectors. Earlier analys is of $X-$ ray emission from clusters has usually assumed an isothermal gas (Jones and Forman 1984; Abramapoulos and Ku 1983; Cavaliere and Fusco-Femrano 1978; Mushotzky et al. 1978). In addition to allowing for nonisothermality in the hot gas, our approach makes a minimum of assumptions about the galaxy density profile, the cluster potential, possible unseen matter, or the relative importance of the various mass components. Though non-isothermal models have had limited application in the past (Cavaliere 1979; Rahcall and Sarazin 1978; White and Silk 1980; Gorenstein et al. 1979), they have suffered from a lack of generality in their use of an isothermal cluster potential.

The alternative approach used in this paper is to derive the cluster potential and binding mass from the temperature and density profiles which describe the $x$-ray spectrum. The flexibility of the model is justified because we find that the spectra of several rich clusters rule out a gas-binding potential with the form of an isothermal sphere and a mass of $1-2 E 15$ solar masses.

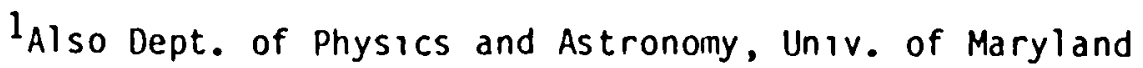




\section{RESIJLTS}

\subsection{The Equation of State of the Gas}

Since the HEAO 1 detectors cover a broad energy band. $(2-60 \mathrm{keV})$ with good spectral resolution (1 keV) they are a useful probe of temperature effects in the $x$-ray emitting gas. Numerical models are generated for a wide range in central temperature and polytropic index (used to parameterize the temperature profile $\left(T / T_{0}=(\rho / \rho)^{\gamma-I}\right)$, where $T_{0}$ is the central temperature and $y$ is the polytropic index. The form of the density profile used in the analysis of the $x$-ray irages $\left(1+\left(r / r_{c}\right)^{2}\right)^{-x}$ where $r_{c}$ is the core radius, is used here. The models are then convolved with the energy response matrix of the detector and $f i t$ to the data. The abundance and the normalization $\left(n^{2} r^{3}\right)$ are free parameters while the column density of absorbing material fn the line of sight is allowed to vary within the levels in Burstein and Heiles (1979). Figure 1 shows the confidence contours for 5 clusters using the combined chisquare for model fits to the spectra $(2-60 \mathrm{keV})$ of both detectors. The 5 clusters presented are those in which the spectra are good enough to meaningfully constrain the equation of state characterized by $r$. An isothermal gas is clearly ruled out for the clusters A1795, A85, and Coma. A2199 is isothermal or nearly isothermal while Perseus is characterized by an intermediate state between isothermal and adiabatic. The spectral parameters are only weakly sensitive to the density profile. Initially we have chosen $x=1.5$, but, the spectra are not strongly sensitive to the exact value of $x$. The effect of a lower $x$ is to make $y$ closer to one (shown for Coma in Figure 2). However, even for a very flat density profile, $x=1.0$ (derived from fitting the $x$-ray surface brightness profile with an isothermal model), for Coma $1.3<\gamma<1.5$. Thus, an isothermal gas is ruled out for Coma with $>90 \%$ confidence.

\subsection{Gas Mass, Binding Mass, and Cluster Potential}

Poissons equation $\nabla^{2} \phi=4 \pi G_{0}$ is used to derive the binding mass distribution from the inferred temperature profile. We assume that the gas is in hydrostatic equilibrium with the cluster potential. The clusters with $4 / 3<\gamma<5 / 3$, imply a potential well much deeper than the truncated isothermal potential used by Bahcall and Sarazin (Figure 3 ) and have about $1 / 5-1 / 10$ the total mass derived from analysis of the galaxies using a king model (Kent and Gunn 1982). Typical binding masses for the polytropes fit here are $2-4 E 14$ solar masses. The ratio of gas mass to binding mass ranges from $22 \%$ for a $\gamma=1.2$, central temperature $=9 \mathrm{keV}$ model to $50 \%$ for a $\gamma=5 / 3$, central temperature $=20$ keV model. Both calculations use parameters appropriate for Coma: a central density of $5 \mathrm{E}-3$, core radius of $.33 \mathrm{Mpc}$, and cluster radius of 3 Mpc. There is a significant uncertainty in the calculation of the gas to binding mass ratio hecause the density profile is not well known, especially outside of $1 \mathrm{Mpc}$, and the core radius is not well determined. He do know that the $X$-ray emission for Coma apparently extends to $3 \mathrm{Mpc}$. However, it is possible that outside of a few core 
radii, the density falls off abruptly and the temperature rises steeply as would be the case if there are heating effects by an intercluster medium (Jones, Forman, and Tucker 1984). This would mean that the gas mass has been overestimated by assuming that the density profile of the gas is described by a one component model.

The resulting gas and gas-binding masses, and the latters distribution is very different from what was previously derived from the optical analysis and isothermal $X$-ray models. The special case of our models, $\gamma=1.08, x=1.5$ well approximates the analytic expression $\left(1+\left(r / r_{0}\right)^{2}\right)-392$ for the derived binding mass distribution. However, the total cluster mass derived by Kent and Gunn for Coma, 1.6E15 solar masses, requires a central temperature of nearly $18 \mathrm{keV}$ in this model. This results from the linear scaling of the binding mass density with

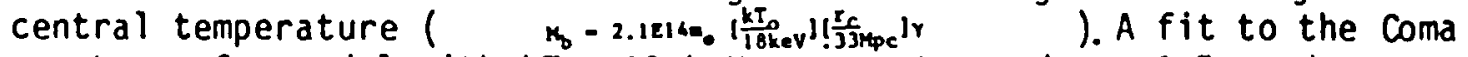
spectrum of a model with $\mathrm{kT}=18 \mathrm{keV}, \gamma=1.08$, and $x=1.5$ produces a reduced chi-square of about 25 .

There has been theoretical and observational evidence against the assumptions that the galaxies are virialized and the unseen binding mass distribution is the same as the galaxies (an isothermal sphere). Recent simulations of galaxy clustering in an open universe by Miller (1984) suggest that clusters are still being assembled, galaxies are continuing to fall in, and that clusters have not yet fully separated out from the Hubble flow. A similar conclusion was reached by Rivolo and Yahil (1983). These studies indicate that the galaxies have not fully responded to the cluster potential, so that the cluster mass determinations which assume the galaxies are virialized overestimate the amount of unseen mass. White's (1976) n-body simulations which associate the entire unseen mass with the galaxies overproduces the amount of luminosity segregation in clusters. This indicates that the galaxy and unseen masses may not have the same distribution. The further specification that these distributions are isothermal spheres is also ooubtful. Geller and Beers (1982) show galaxy contour maps which suggest that few galaxy distributions appear to be isothermal spheres, and that many show asymmetries and substructure. We found (Henriksen and Mushotzky 1984) that the models for X-ray emission based on the assumptions detailed above produced significant inconsistencies between $x$-ray and optical determinations of the characteristic parameters.

On the other hand, surface brightness maps of many rich clusters appear to be smooth and relatively symmetric suggesting that the gas has evolved in the potential well of the cluster. The condition for virialization of the cluster gas $\left[\frac{\mathrm{kT}}{0.2 \mathrm{kev}}\right]>>\left[\frac{\mathrm{H}_{\mathrm{O}}}{100^{\circ}}\right]\left(\frac{\mathrm{M}}{10^{14}} \mathrm{~m}_{0}\right]^{1 / 2}\left[\frac{\mathrm{R}}{\mathrm{Mpc}}\right]^{1 / 2}$ is

\subsection{Iron Abundance}

The Fe abundance is determined using the atomic data of Raymond and Smith (1979) for a plasma in collisional equilibrium. The iron lineblend involving transitions in Fe $X X V$ and FeXXVI is the primary feature in the spectrum and occurs at an unredshifted energy of $6.7 \mathrm{keV}$. There is evidence also of a marginal detection of the $K_{B} l i n e-b l e n d$ at $7.8 \mathrm{keV}$ in several clusters (Figure 4). Typically, the abundances are .5 solar 
(Mushotzky 1984), though a few clusters, most notably Coma, indicate an abundance substantially below this value (Figure 5).

\section{DISCUSSION}

Non-isothermality in the cluster gas is not surprising since thermal conduction would probably be inhibited by the "galactic" magnetic fields present in the cluster. This is consistent with the suggestion by Pryor and Geller (1984) that the galaxy velocities in Coma are isotropic out to 7 core radii $(2.5 \mathrm{Mpc})$, if we assume that the magnetic fields are tied to the galaxies. All of the clusters we found which were consistent with $\gamma=5 / 3$ were also consistent with intermediate values of $\gamma$. We note that the density profiles derived from modeling the $X$-ray surface brightness profiles with isothermal models generally give a density profile of $1.00<x<1.25$. If these values of $x$ are used, an intermediate value of $\gamma$ is favored. The intermediate values of $\gamma$ might result from a central isothemal region surrounded by an adiabatic region. An isothermal region might be brought about by thermal conduction if the magnetic fields were weak enough in the central region. The theoretical simulations of clustering suggest that few galaxies have traveled through the cluster center more than once. This would also indicate that the recently observed radial gradients in the HI content of spiral galaxies in Coma (Bothun et al. 1984) are probably due to shorter gas depletion times rather than ram-pressure stripping (Kennicut et al. 1984). This might also lead to the metal deficient gas observed in Coma. Alternatively, isothermality in the central region may be brought about by the cooling effect of gas injection by the galaxies. Since $50 \%$ of the total surface brightness comes from the inner 2 core radii in Coma, the cooling effect would have to confined to this central region. The theoretical work relevant to this has not been done. Cowie and Binney (1977) produced a radiation regulated gas flow model which combined many of the physical mechanisms suggested here. The model used an isothermal mass distribution for the cluster binding mass. We believe that this accounts for the nearly flat temperature profiles the model predicts which fail to match the observed spectrum at low energies. Dur work shows that this results from the isothermal assumption. The HEAO 1 spectrum $(2-60 \mathrm{keV})$, which extends to higher energy than the spectrum $(2-10 \mathrm{keV})$ used in their paper, also shows emission at higher energies which the isothermal profile cannot account for.

\section{SUMMARY}

It is obvious that there are many variables which affect the observed $X$ ray properties of clusters. However, the optical and $X$-ray observations together suggest a cluster with basic parameters which are very

different from the previous assumptions. In particular, the assumptions of a King potential, virialization of the galaxies, and isothermality of the hot intracluster medium may not he valid. Therefore, previnus calculations of the total cluster mass may be incorrect. 


\section{REFERENCES}

Abramapoulos, F., and Ku, W.H.-M. 1983, Ap. J., 271, 446.

Bahcall, J.N., and Sarazin, C.L. 1978, Ap.J., 219, 781.

Bothun, G.D., Schommer, R.A., and Sullivan, W.T. 1984, Astron. J., 89, 466.

Burstein, D., and Heiles, C. 1982, Astron. J., 87, 1165.

Cavaliere, A. 1979, in X-ray Astronomy, (eds) R. Giacconni and G. Setti, Dordrecht:D. Reidel.

Cavaliere, A., and Fusco-Femiano, R. 1978, Astron. and Astrophys., 70, 677.

Cowie, L.L., and Binney, J. 1977, Ap. J., 215, 723.

Forman, W., Jones, C., and Tucker, W. T984, Ap. J., 277, 19.

Geller, M.J., and Beers, T.C. 1982, PASP, 94, 421.

Gorenstein, P., Fabricant, D., Topka, K., Harnden, F.R. jr. 1979, Ap. J., $230,26$.

Gunn, J.E., and Gott, J.R. 1972, Ap. J., 176, 1.

Henriksen, M.J., and Mushotzky, R.F. 1985, Ap. J., in press.

Jones, C., and Forman, W. 1984, Ap. J., 276, 38.

Kennicutt, R.C. jr, Bothun, G.D., Schommer, R.A. 1984, Astron. J., 89, 1279.

Kent, S.M., and Gunn, J.E. 1982, Astron J., 87, 945.

Lea, S.M., Silk, J.1., Kellogg, E., and Murray, S.S. 1973, Ap. J. (letters), 184, L105.

Miller, R.H. 1984, Astron. and Astrophys., 138, 121.

Mushotzky, R.F., Serlemitsos, P.J., Smith, B.W., Boldt, E.A., and Holt, S.S. 1978, Ap. J., 225, 21.

Mushotzky, R.F. 1984, Physica Scripta, V.T7.

Pryor, A. and Geller, M.J. 1984, Ap. J., 278, 457.

Raymond, J.C., and Smith, B.W., 1979,(private communication)

Rivolo, A.R., and Yahil, A. 1983, Ap. J., 274, 474.

White, S.D. 1976, MNRAS, 177, 717.

White, S.D., and Silk, J., 1980, Ap. J., 241, 864. 

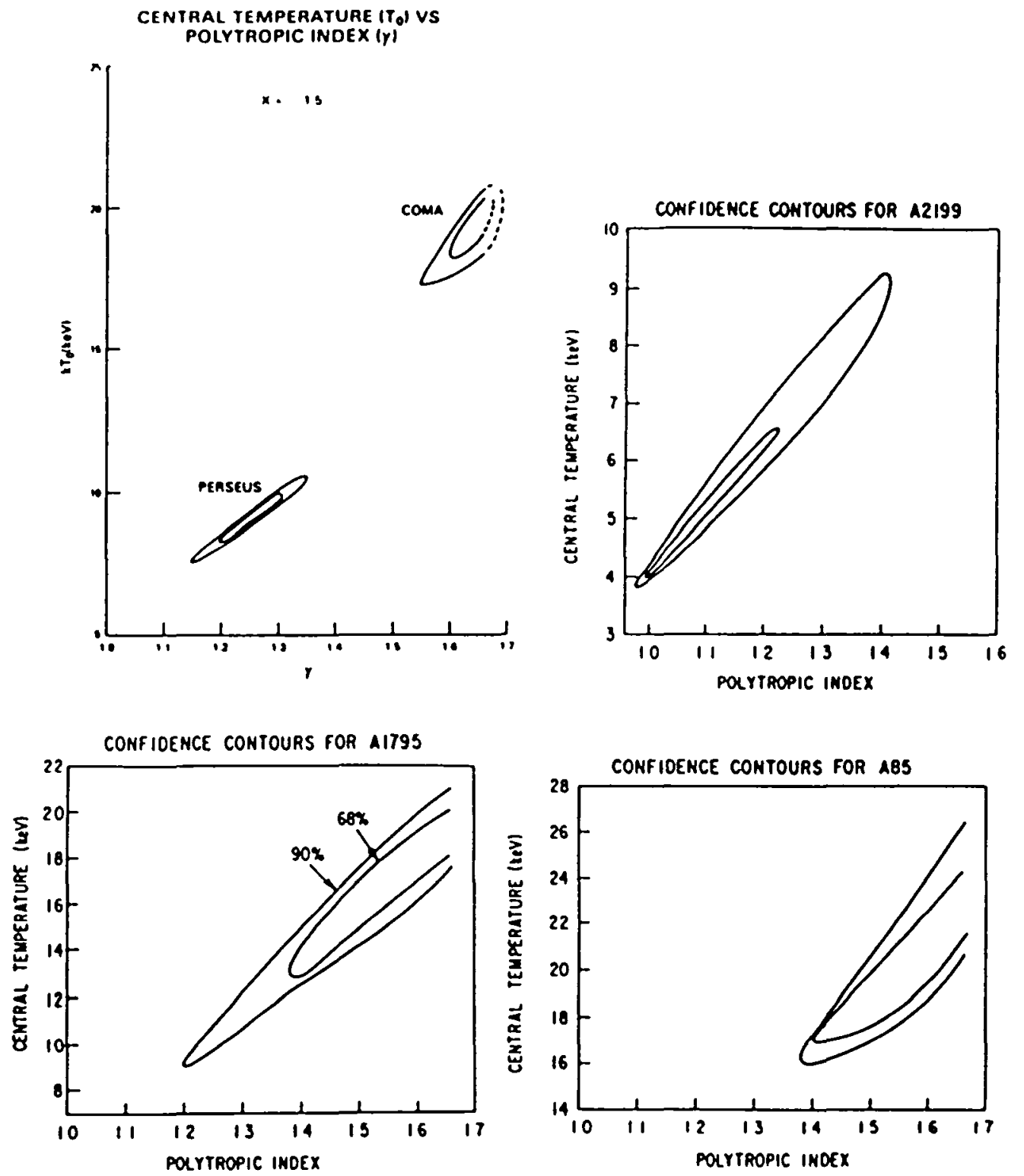

Figure 1: Confidence contours for A85, A1795, and A2199 are derived from fitting models to the MED and HED data. Contours for the Coma and Perseus clusters result from fitting the MED data. The density parameter, $x$, is 1.5 for all clusters. 

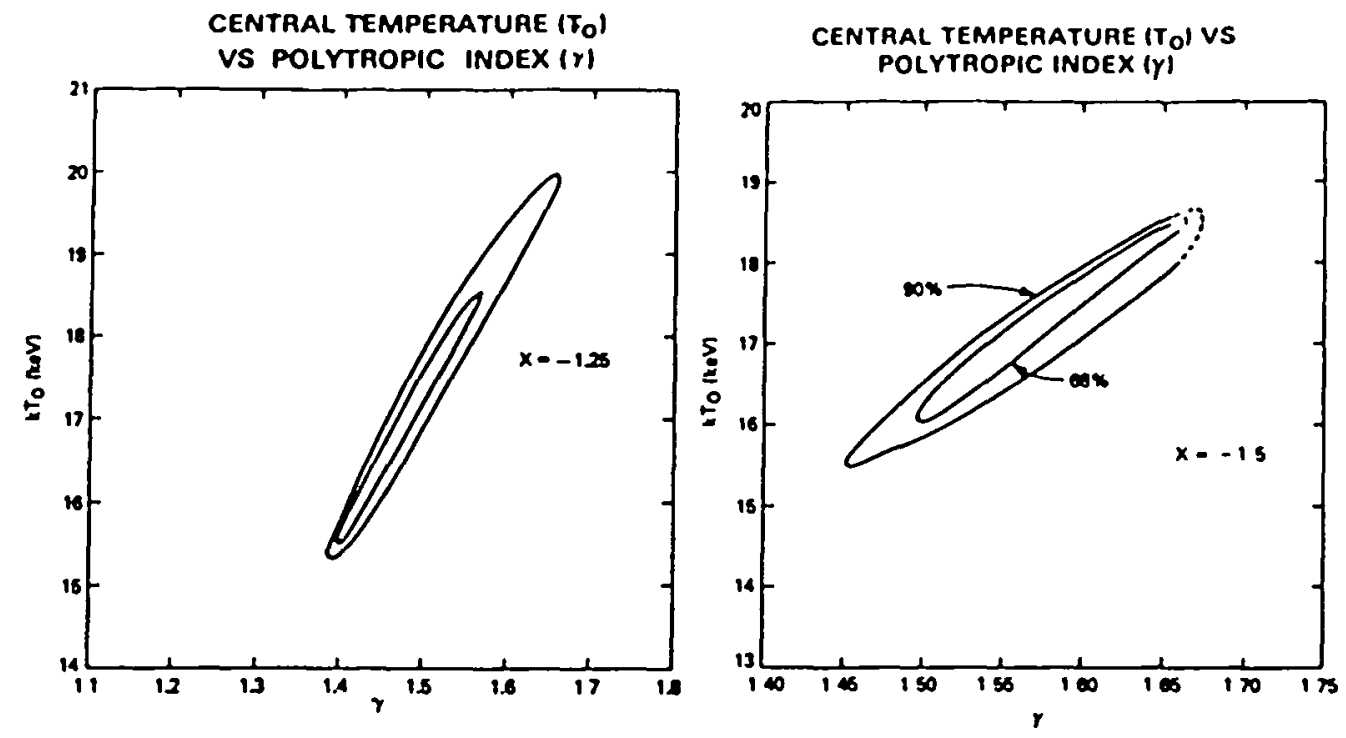

Figure 2: Confidence contours for the coma cluster are shown for 2 density profiles of the form $\left.(1+R / a)^{2}\right)^{-x}$. Models are fit to the MED and HED data. Comparison with Fig. 1 demonstrates consistency between detectors.

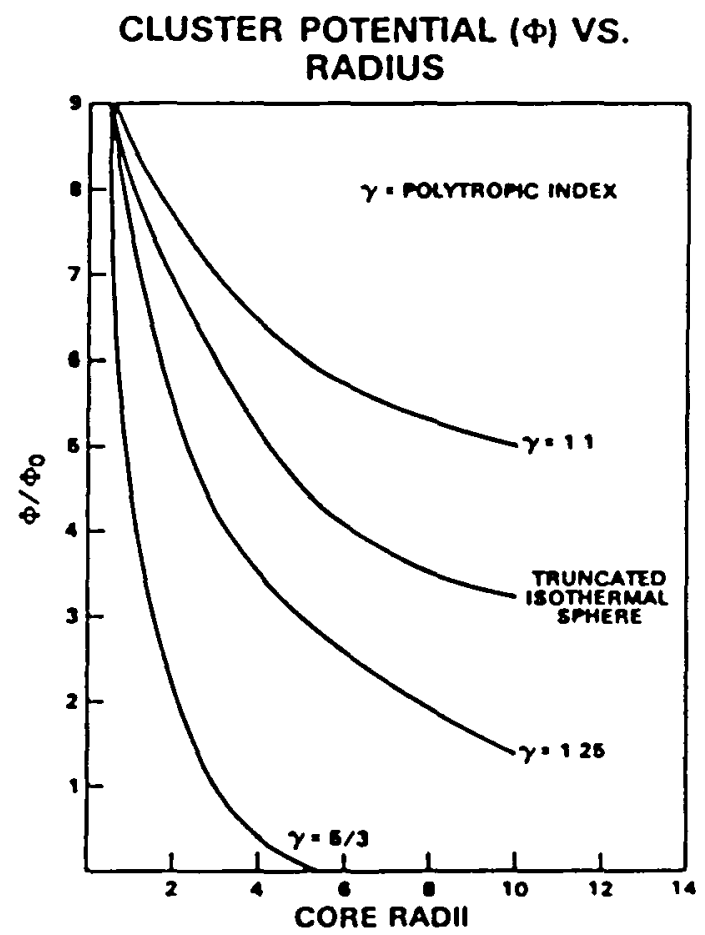

Figure 3: Potentials are derived for a range in polytropic index with $x$ $=1.5$. The nearly isothermal potential used in earlier polytropic models is given for comparison. 


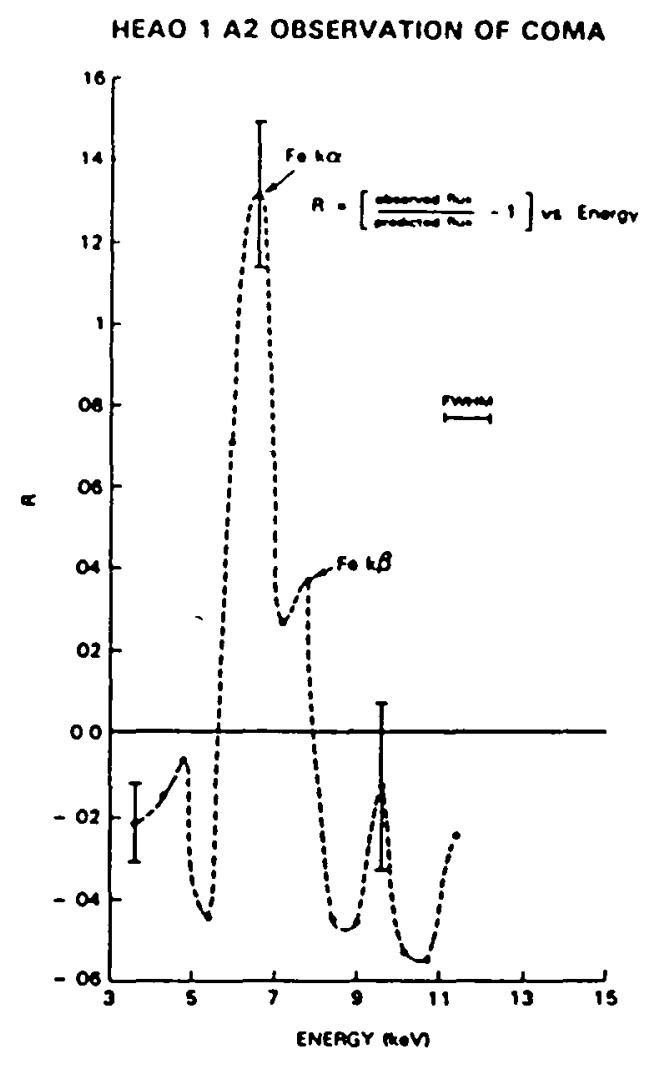

Figure 4: Residuals are plotted for a thermal bremsstrahlung continuum fit to the MED data. A strong detection of the Fe $K$ line blend and a possible weak detection of the $\mathrm{Fe} K_{B}$ line blend are fisible.

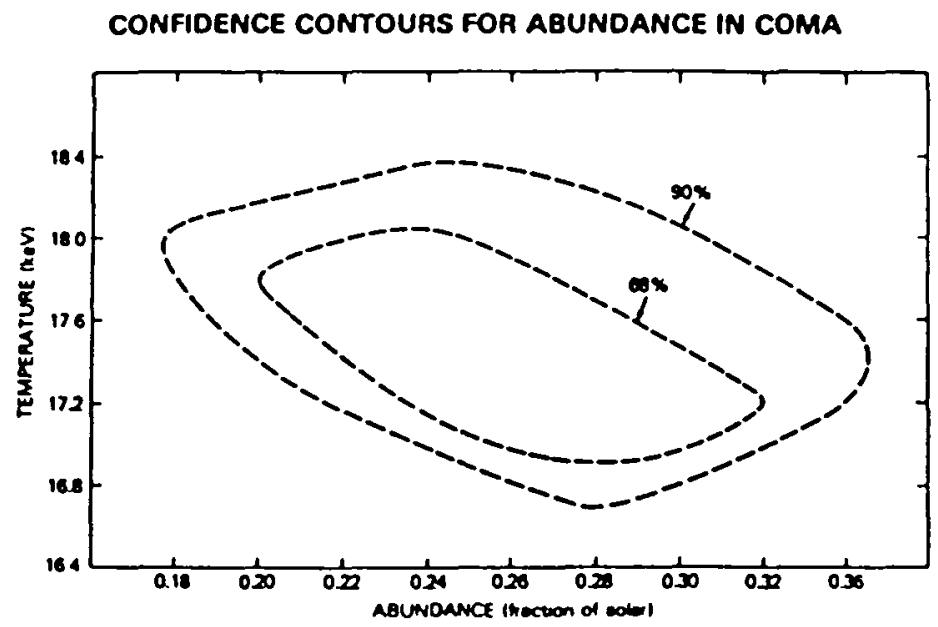

Figure 5: Confidence contours of central temperature vs. abundance are. shown for the Coma cluster. 


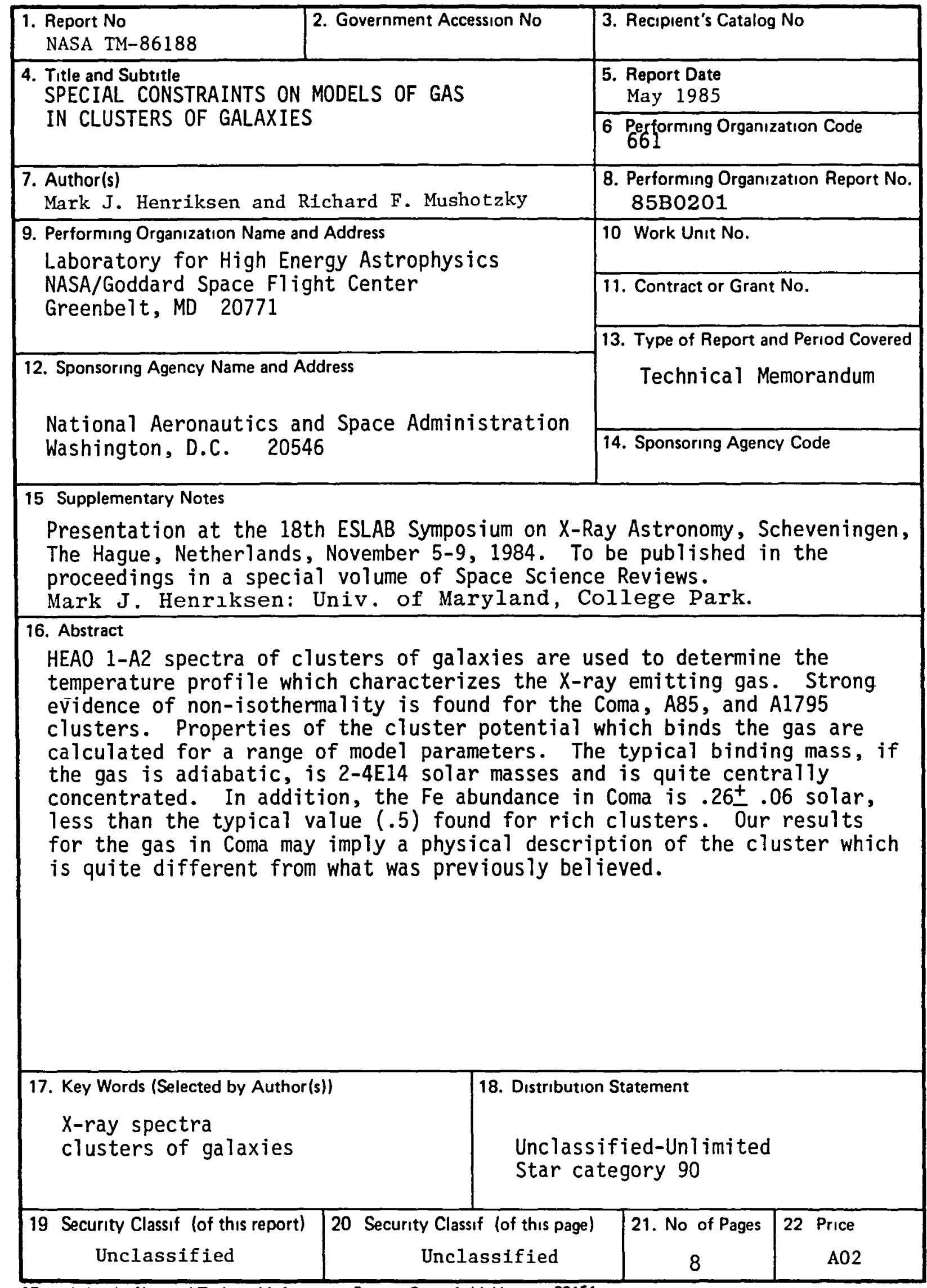

- For sale by the Natıonal Technical Information Service, Springfield, Virginıa 22161 


\section{End of Document}

